\title{
Determination of Rod's Position by the Shadow
}

\author{
Saishuo Zheng ${ }^{1, \text { a }}$ \\ ${ }^{1}$ School of North China Electric Power University, Baoding 071000, China; \\ a591039822@qq.com
}

Keywords: Shadow positioning, Particle swarm optimization algorithm, Exhaustive method

\begin{abstract}
To find out the position of the rod with its shadow, a model for determining the position of the straight rod with the objective function of minimizing the error value is established. Some results had been obtained by particle swarm optimization algorithm, and were classified by the system cluster analysis to achieve the optimal results. After that, the optimal results were tested.
\end{abstract}

\section{Introduction}

How to determine the shooting location and the shooting data of a video film is an important aspect of video data analysis, shadow position technology is a method to determine the location and date of the video shoot by analyzing the changes of objects' shadow.

To find out the position of the rod with its shadow, considering the regularity of the moving position of the straight rod's shadow, that is, its position of the moving is almost a straight line, which can be regarded as a horizontal plane near the position of the straight rod.

In order to make the obtained position of the rod can be as much as possible to meet the problem of data requirements, select the results that make the residual sum of squares of values of existing data as the object function and make the requirements based on latitude and time the constraint conditions.

Based on the analysis above, there are two methods has been proposed.

\section{The method}

Calculating the shadow length of the straight rod, the length is found to be linear with time. That is, the position of the rod can be approximately regarded as the horizontal space. The picture is shown as follows.

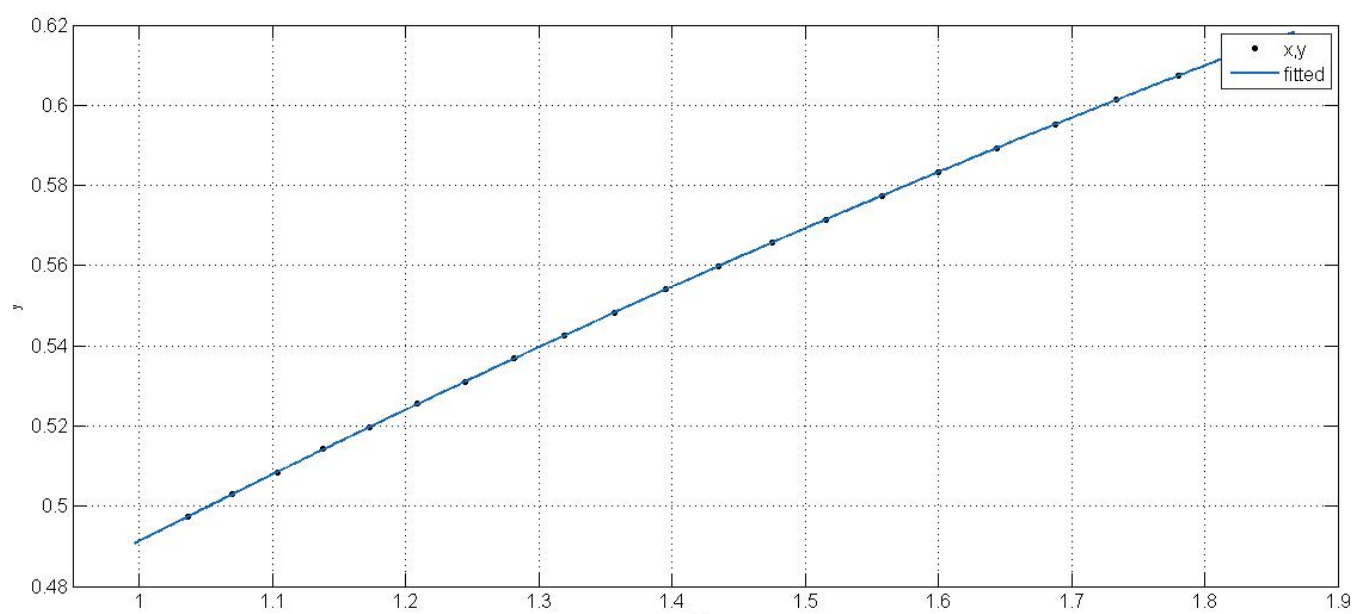

Fig 1: Shadow positioning figure

As a result, according to the length of the shadow and the relation of the given coordinate values, we can deprive:

$$
L=\sqrt{x^{2}+y^{2}}
$$

As for the relationship between the length of the rod and the shadow: 


$$
\tan h=\frac{H}{L}
$$

Combined the equation two with the basic knowledge of Geography, we can know that:

$$
\begin{gathered}
L=H \cdot \frac{1}{\tan (\arcsin (\sin \varphi \sin \delta+\cos \varphi \cos \delta \cos \tau))} \\
\tau_{i}=\tau_{0}+i \times \Delta \tau, i=0,1,2, \ldots, 20
\end{gathered}
$$

In the equation above, $\tau_{0}$ shows the local time when the Beijing time is $14: 42, \Delta \tau$ means the time angular difference of 3min.

In order to make the obtained position of the rod can be as much as possible to meet the problem of data requirements, the objective function of minimizing the error value is set up. Integrated the actual situation about the latitude and time:

$$
\begin{gathered}
\min f=\sum_{i=0}^{20}\left(\hat{L}-\sqrt{x^{2}+y^{2}}\right)^{2} \\
\text { s.t. }\left\{\begin{array}{c}
-\frac{\pi}{2}<\varphi<\frac{\pi}{2} \\
-\frac{\pi}{2}<\tau<\frac{\pi}{2} \\
H>0
\end{array}\right.
\end{gathered}
$$

Because the expression of the $\hat{L}$ is so difficult, MATLAB can't fit the appropriate $\varphi, H$ and $\tau_{0}$. Search based on particle swarm optimization algorithm, and use the local information and global information to guide the search; easy to fly over the local optimal information; quick convergence and other advantages, choosing the particle swarm optimization algorithm to calculate the global optimal solution of the particle swarm.

In the basic particle swarm optimization algorithm, the influence of the parameters on the optimal performance of the algorithm is very significant. As a result, we take the reference value given by the scholars of academic circles Carlisle and set the particle number to 200 to expand the search scope to obtain the global optimal solution more accurately. On this basis, using MATLAB to solve the multi particle swarm algorithm can get the results shown in the following table:

\begin{tabular}{ccccccccccc}
\hline group & 1 & 2 & 3 & 4 & 5 & 6 & 7 & 8 & 9 & 10 \\
\hline latitude (rad) & 0.38 & 0.35 & 0.38 & -0.15 & -0.1 & 0.39 & 0.36 & -0.1 & -0.1 & -0.1 \\
Angle (rad) & 0.41 & 0.47 & 0.39 & 0.45 & 0.59 & 0.38 & 0.44 & 0.5 & 0.6 & 0.628 \\
\hline group & 11 & 12 & 13 & 14 & 15 & 16 & 17 & 18 & 19 & 20 \\
\hline latitude (rad) & 0.3834 & 0.3793 & 0.3029 & -0.1774 & 0.3 & -0.1181 & 0.2511 & 0.3105 & 0.3927 & 0.2414 \\
Angle (rad) & 0.3733 & 0.4033 & 0.6251 & 0.3058 & 0.5744 & 0.567 & 0.6446 & 0.672 & 0.3262 & 0.6796 \\
\hline
\end{tabular}

Table 1: The results of particle swarm optimization

The corresponding latitude and longitude can be derived from the 20 sets of data. Using SPSS make a system clustering for the 20 sets data, the results are shown as: 


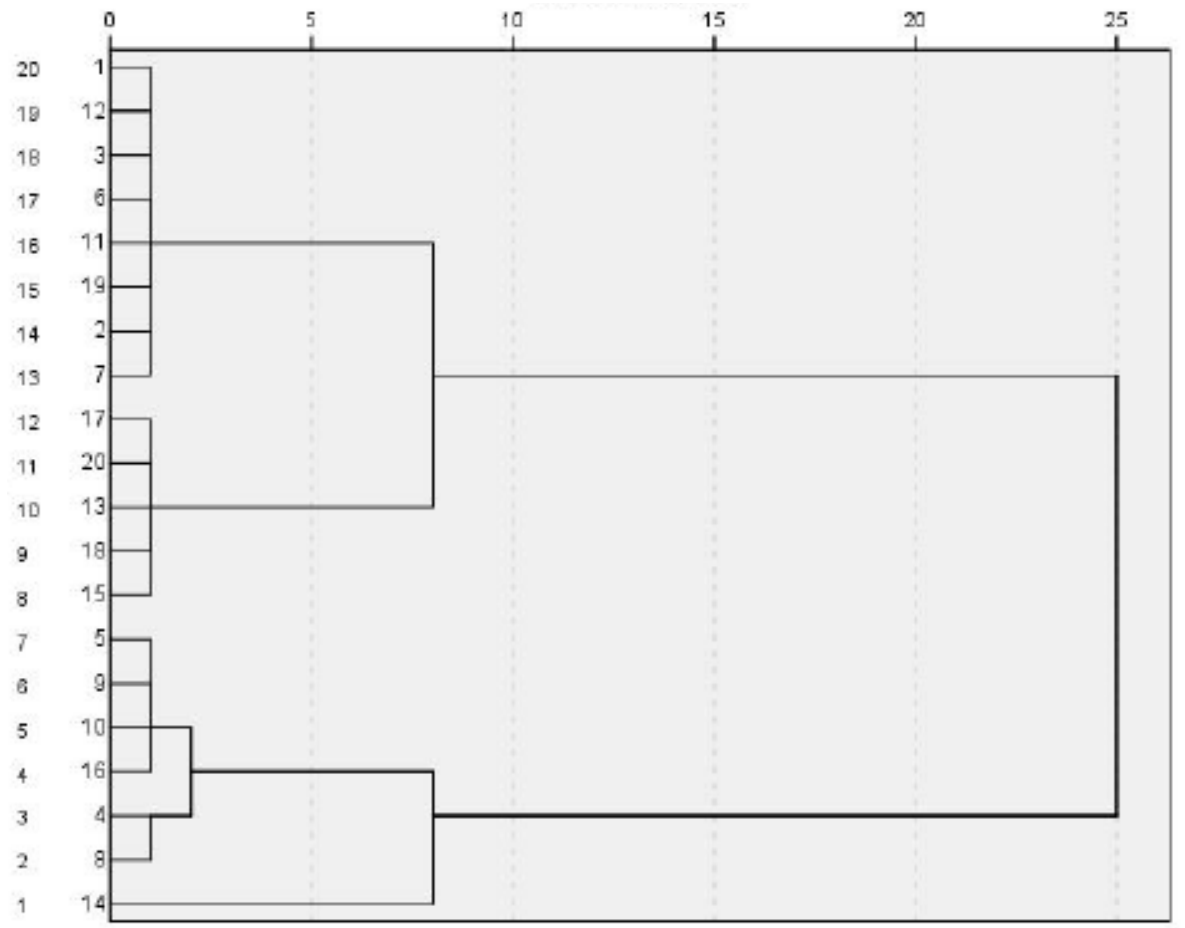

Fig 2: The results of system clustering

According to the picture above, the data can be divided into three groups. To achieve a more actual answer, selecting the position that the sum of the distance between all of the position in the three groups is shortest as the results. After calculating by LINGO, we can get three different result, they are:

\begin{tabular}{ccc}
\hline group & longitude & latitude \\
\hline one & $102^{\circ} 20^{\prime} 20^{\prime \prime}$ & $21^{\circ} 37^{\prime} 33^{\prime \prime}$ \\
two & $116^{\circ} 17^{\prime} 45^{\prime \prime}$ & $16^{\circ} 6^{\prime} 36^{\prime \prime}$ \\
three & $112^{\circ} 27^{\prime} 6^{\prime \prime}$ & $6^{\circ} 22^{\prime} 48^{\prime \prime}$ \\
\hline
\end{tabular}

Table 2: the results of the rod position

To know the specific geographic information of the rod position, using Google maps to view the actual coordinates, can know the possible position were Laos, South China Sea and Indonesia.

In order to test the correctness of the results, take the high angle of the sun at noon and the correlation between the results of each test method to verify the accuracy of the results of the straight rod position. At noon:

$$
\sin h=\sin \delta \sin \varphi+\cos \delta \cos \varphi \cos \tau
$$

That is:

$$
h=\frac{\pi}{2}-|\delta-\varphi|
$$

According to the calculation of the straight rod position determination model, the longitude, latitude and the length of the rod have been obtained. On the basis of providing the correlation of midday sun angle, altitude and latitude to carry out to verify the accuracy of the model results. Using MATLAB to test the result of the position of the straight rod, we find out the group two and the group three in the results are meet the requirements of the reality. 


\section{Summary}

From the search process of the rod position, we find that particle swarm optimization algorithm model can not only optimize the objective function to get more actual and satisfying results, but also can avoid the time-consuming problem of traversal method.

\section{References}

[1] Keke Zheng, Xiaoyong Chen. Discussion on the formula of the sun's red and green in the calculation of building sunlight [J]. Zhejiang architecture, 2011, 28 (9): 6-8.

[2] Lin Lin. Using the calculation of the solar coordinates to carry out the high measurement and positioning [J]. Journal of Nanjing Forestry University, 1991, 15 (3): 89-93.

Reference to a book:

[3] Shoukui Si. Mathematical modeling algorithm and application [M]. National Defence Industry Press, 2011

[4] Qiyuan Jiang, Jinxing Xie, ye Jun. Mathematical model [M]. Higher Education Press: Beijing, 2011:422-423. 